Proceedings of the ASME 2019

International Design Engineering Technical Conferences and Computers and Information in Engineering Conference

IDETC/CIE2019

August 18-21, 2019, Anaheim, CA, USA

DETC2019-97642

\title{
ANALYZING CUSTOMER NEEDS OF PRODUCT ECOSYSTEMS USING ONLINE PRODUCT REVIEWS
}

\author{
Jackie Ayoub \\ Department of Industrial and Manufacturing \\ Systems Engineering, \\ University of Michigan, Dearborn \\ Dearborn, Michigan, 48128 \\ Email: jyayoub@umich.edu

\section{Qianli Xu} \\ Image and Video Analytics Department \\ Institute for Infocomm Research, \\ 1 Fusionopolis Way, \#21-01 Connexis, \\ Singapore, 138632 \\ Email: qxu@i2r.a-star.edu.sg
}

\author{
Feng Zhou \\ Department of Industrial and Manufacturing \\ Systems Engineering, \\ University of Michigan, Dearborn \\ Dearborn, Michigan, 48128 \\ Email: fezhou@umich.edu \\ Jessie Yang \\ Department of Industrial and Operations \\ Engineering, University of Michigan, Ann Arbor \\ Ann Arbor, Michigan, 48109 \\ Email: xijyang@umich.edu
}

\begin{abstract}
It is necessary to analyze customer needs of a product ecosystem in order to increase customer satisfaction and user experience, which will, in turn, enhance its business strategy and profits. However, it is often time-consuming and challenging to identify and analyze customer needs of product ecosystems using traditional methods due to numerous products and services as well as their interdependence within the product ecosystem. In this paper, we analyzed customer needs of a product ecosystem by capitalizing on online product reviews of multiple products and services of the Amazon product ecosystem with machine learning techniques. First, we filtered the noise involved in the reviews using a fastText method to categorize the reviews into informative and uninformative regarding customer needs. Second, we extracted various customer needs related topics using a latent Dirichlet allocation technique. Third, we conducted sentiment analysis using a valence aware dictionary and sentiment reasoner method, which not only predicted the sentiment of the reviews, but also its intensity. Based on the first three steps, we classified customer needs using an analytical Kano model dynamically. The case study of Amazon product ecosystem showed the potential of the proposed method.
\end{abstract}

\section{INTRODUCTION}

\subsection{Background}

Product ecosystem includes multiple related products manufactured by the same company to achieve superior customer satisfaction and user experience. Examples of product ecosystems include what Apple, Amazon, or Samsung offers. The success of these product ecosystems depends more on the overall user experience within the product ecosystem rather than the performance of single products or services. This is probably due to the fact that, within a product ecosystem, a main product is supported by other products and services to create customer satisfaction and user experience that other single offerings cannot beat [1]. In addition, once a customer enters such an ecosystem, it will be hard to exit due to the high cost of transferring the applications to other devices [2].

Although we have witnessed the success of product ecosystems, it is often challenging to create a successful product ecosystem from the perspective of design. Previous researchers have attempted to address different issues of product ecosystem design. For example, Levin et al. [3] created a framework for designing user experience of multiple devices within a product 
ecosystem, including smartphones, tablets, TVs, and computers, based on consistent, complementary, and continuous techniques. Gawer and Cusumano [4] discussed the impacts of internal (or company-specific) and external (or industry-wide) platforms on product innovation. Internal platform promoted innovation by considering the derivative of products and services while the external platform considered complementary products and services for an innovative ecosystem. Jiao et al. [5] incorporated the notion of ambience or context of human-product interaction in product ecosystem design in order to enhance user experience. $\mathrm{Oh}$ et al. [6] presented a product-service system design framework within a business ecosystem, including manufacturers, suppliers, and content providers, to identify design factors for products and services. Zhou et al. [1] presented a three-stage product ecosystem design process for user experience. The design stages included affective-cognitive need acquisition, affective-cognitive analysis, and affective-cognitive fulfillment. Their results showed that high-level needs, including affective and cognitive needs, can improve user experience. However, the framework was mainly conceptual and no concrete research methods were provided for each step. Later, in order to show such a design process, Zhou et al. [7] described the relations between user experience and the components of a product ecosystem using a simulation technique named fuzzy reasoning Petri nets. However, such a simulation method may be restricted in terms of its generalizability. In this paper, we attempted to analyze various customer needs associated with different products and services within a product ecosystem with a concrete case study. While it is relatively easy to elicit and analyze customer needs of single products, it is challenging to do for a whole product ecosystem.

\subsection{Research Challenges and Suggested Solutions}

1) Collecting Customer Need Data: There are various products and services involved in the product ecosystem. Hence, it is time-consuming to collect data in order to elicit customer needs using traditional methods, such as interviews, focus groups, and surveys [8], compared to the short product development lead time [9]. Recently, many researchers have made use of online-user generated data (e.g., online product reviews) to identify customer needs. For example, Zhou et al. [10] extracted customers' latent and explicit needs based on online product reviews using case analogical reasoning and sentiment analysis. For one thing, users describe the product performance in various use situations from the users' perspectives in these online product reviews, which provide a good channel for customer needs elicitation [11]. For another, the scale of the online user-generated data is often so large that it can extract the customer needs associated with the products and services. For example, Amazon Echo Dot 2 has more than 120,000 product reviews on Amazon.com. Therefore, in this research, we will make use of the online user-generated data to identify customer needs.

2) Analyzing Text Data: Customer needs identification and analysis are ambiguous since they are written in the form of natural languages [12]. It often needs domain experts to analyze customer needs with specific skills. For example, experts who can conduct ethnographic studies can have a deep understanding of customer needs. However, it is extremely time-consuming to analyze such a large amount of text data, if not impossible [13]. In order to reduce the ambiguity of customer needs involved in the text data and analyze text data efficiently and effectively at the same time, we propose to make use of machine learning methods in this research.

First, we cleaned the data by filtering out the noise involved in the textual review data. In order to do that, we proposed to employ a supervised machine learning technique, fastText [14], to distinguish informative reviews from uninformative reviews (i.e., noise) with regard to customer needs. For example, the review, "I love this e-reader" is not informative in terms of customer needs analysis since it does not show what specific customer need is satisfied, although it shows a positive attitude towards the product. fastText is a supervised machine learning technique, and it is reported to be an order of magnitude faster in performance than the state-of-the-art deep learning classifiers, but is as accurate as them [14]. Such a step improves data quality greatly and reduces the ambiguity of customer needs embedded in noisy text data.

Second, in order to identify different topics of customer needs within the product ecosystem, we proposed to apply a topic modeling technique, latent Dirichlet allocation (LDA). LDA is an unsupervised machine learning technique and it assumes that each review is a mixture of a small number of topics related to customer needs and that each word's presence is attributable to one of the review's topics [15]. Therefore, LDA can identify the hidden topics, indicating the voices of customers from a large volume of unstructured online product reviews [16]. Each topic identified is related to a specific customer need for individual products and/or services within the product ecosystem expressed in product reviews. The topics identified can categorize customer needs into different groups, which are then combined with the quantitative customer preferences (i.e., satisfaction and dissatisfaction score of each topic) as input for categorizing customer needs for the product ecosystem so that the superiorities and weaknesses of product ecosystem can be discovered.

Third, in order to quantitatively understand to what extent customers are satisfied with the products and services, we conducted sentiment analysis of each product review associated with each group of customer needs to pave the way for customer needs categorization in the next step. Sentiment analysis is a computational method to predict whether a product review is positively, neutrally, or negatively evaluated [17]. Researchers have proposed various machine learning methods for the purpose of sentiment analysis, including both supervised and unsupervised methods. For example, Hu and Liu [18] compiled a list of lexicon seeds (both positive and negative ones), which were expanded using synonym and antonym relations in WordNet. Ding et al. [19] created a holistic lexicon list by exploiting external evidence and linguistic rules in the language expressions, which outperformed the one in [18]. Chen et al. [20] built a sentiment analysis model with conditional random fields using various linguistic features, including part-of-speech tags and lexicons, and obtained accuracy over $75 \%$ in terms of the $F_{1}$ measure. Kim [21] proposed a deep learning model based on word embeddings and convolutional neural networks, and the prediction accuracy was between $81.5 \%$ and $93.4 \%$ across 
various datasets. Li et al. [22] trained an adversarial memory network for sentiment analysis across different domains (e.g., product reviews vs. movie reviews) and visualized the pivotal words in the review to improve the interpretability of their deep model. Although these sentiment analysis methods classified whether the review belonged to the positive, the negative, or the neutral category, they failed to give an intensity score, which is necessary in this research to quantitatively identify the satisfaction and dissatisfaction scores that are used to categorize customer needs in the next step. In order to do this, we proposed to employ VADER (Valence Aware Dictionary and sEntiment Reasoner) as an unsupervised method to predict customer satisfaction quantitatively. Hutto and Gilbert [23] has shown that this method was able to outperform human raters in terms of the $F_{1}$ measure, especially for social media text data.

3) Categorizing Customer Needs: It is necessary to categorize customer needs into different groups in terms of their priority and importance in creating great user experience and improving customer satisfaction. Many techniques have been proposed in the literature in this aspect [24]. For example, the Kano model is widely used to identify four basic drivers of customer satisfaction, i.e., must-be, one-dimensional, attractive, and indifferent [25]. Kim and Han [26] identified three usability dimensions, including product, product-user, and product-usertask, in order to evaluate customer satisfaction in terms of usability. Delin et al. [27] classified customer needs into three groups, i.e., highest, medium, and least concept coverage, in order to identify the most appropriate emotional adjectives in describing different customers. However, the methods mentioned above only offered a qualitative evaluation of customer satisfaction regarding product performance level [28]. In order to make use of quantitative measures to evaluate user experience and satisfaction, it is important to assign customer satisfaction and dissatisfaction scores. In this research, we proposed to use an analytical Kano model [30]. It is consistent with the Kano principles, but incorporates quantitative measures produced from sentiment analysis in the previous step into customer satisfaction, which can be used as the tangible criteria for categorizing customer needs.

\section{PROPOSED METHOD AND CASE STUDY}

In this work, we presented a method to analyze customer needs of the Amazon product ecosystem based on its online customer reviews to understand to what degree Amazon products and services are satisfying their customers. The Amazon product ecosystem (services and products) is used to illustrate the proposed method. We collected a total number of 91738 review sentences between 2011 and 2018 for the following products: Amazon Kindle Fire tablets, Kindle Ereader, Fire TV, Echo and Alexa devices, and other accessories (e.g., Kindle keyboard, Kindle leather cover, Fire TV power adapter, USB chargers). There are four important steps involved in the proposed method as illustrated in Figure 1, including 1) noise filtering, 2) topic extraction, 3) sentiment analysis, and 4) categorizing customer needs. The noise filtering step applies the fastText method to remove uninformative reviews from the informative reviews in order to reduce the ambiguity involved in customer needs. The topic extraction step uses the LDA method to identify the underlying topics associated with the customer needs of individual products and services within the Amazon product ecosystem. The sentiment analysis step makes use of the VADER method to not only predict sentiment polarity, but also sentiment intensity scores of product reviews in order to quantitatively evaluate customer satisfaction and dissatisfaction regarding each customer need produced in the previous step. Finally, the analytical Kano model is used to classify customer needs based on the satisfaction and dissatisfaction scores produced in the previous step as the tangible criteria. According to the obtained results from this study, possible design recommendations can be made to improve user experience and customer satisfaction. Such a process is iterative in order to continuously improve the performance of the product ecosystem.

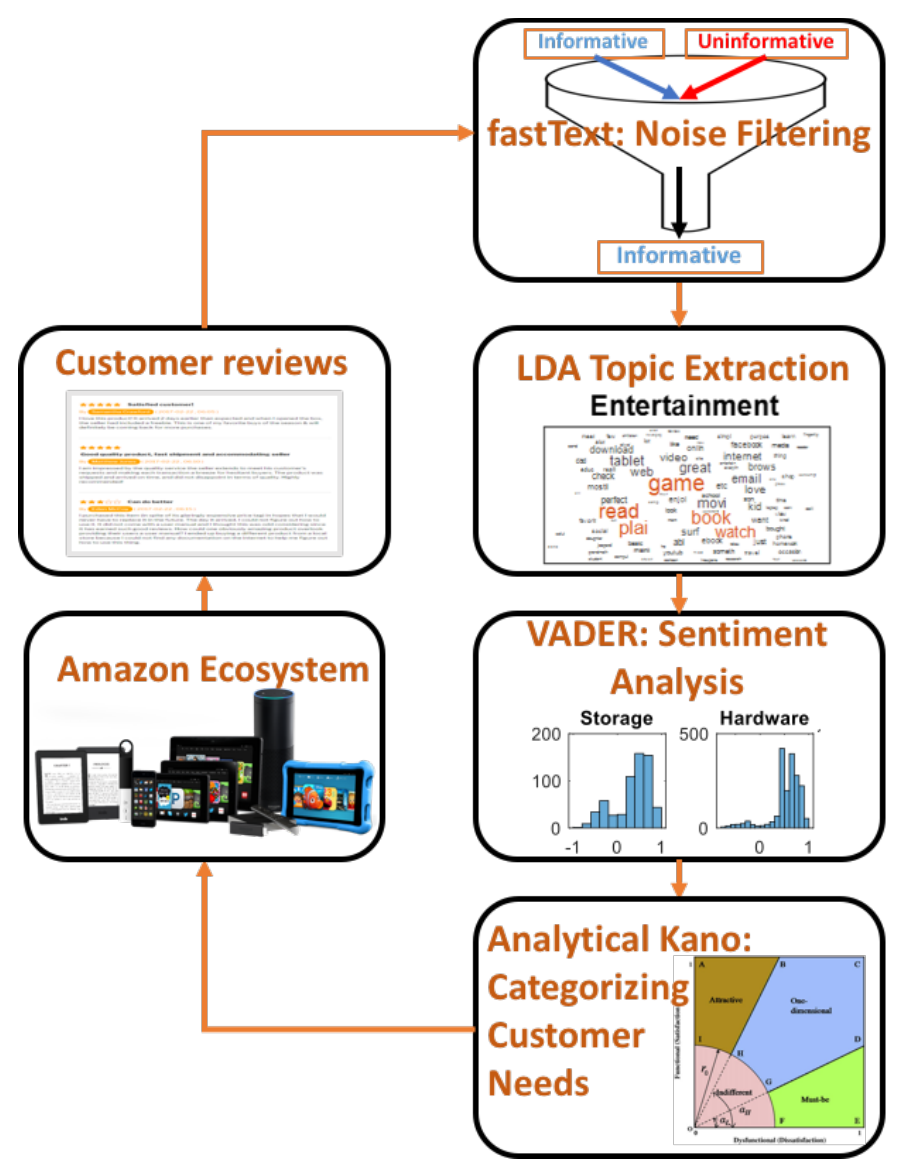

\section{FIGURE 1: THE STEPS INVOLVED IN THE PROPOSED METHOD}

\section{FILTERING NOISE USING FASTTEXT}

The fastText method is a supervised machine learning technique created by Facebook for text classification and word vector representation. fastText assumes that words are formed by a $n$-grams of characters where $n$ can range from 1 to the length of the word. Compared to word2vec and glove, fastText can find a vector representation for rare words not present in the dictionary by breaking the words into chunks of vectors and combining them to create the final vector, which is particularly useful for text data in social media [19]. 


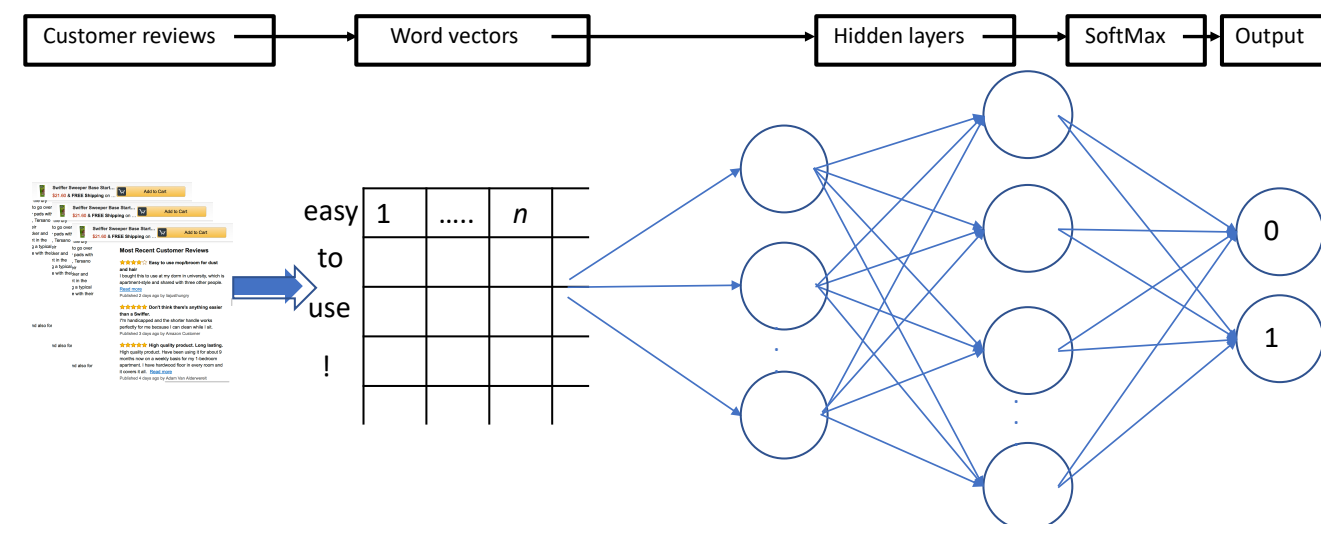

FIGURE 2: THE MODEL OF FASTTEXT

The procedure of noise filtering using fastText is shown in Figure 2. In order to train and test the model, 10,000 reviews were manually labeled into informative (7453 labeled as 1) and uninformative (2543 labeled as 0 ) reviews and 4 were removed as they were not in English or blank. By uninformative reviews we mean that they do not describe specific features of the product or provide only a general opinion on the product. Thus, they are not helpful to elicit customer needs (e.g., "I have 3 echoes and 2 dots", "Very pleased", and "This was my first tablet..."). The next step was to preprocess the reviews, including removing punctuations, converting the letters into lowercase, and stemming.

Furthermore, by varying the fastText parameters, including the learning rate, number of epochs, dimension of word embeddings, and number of $n$-grams and using a 5-fold crossvalidation, we obtained the following results: precision $=0.91$, recall $=0.93$, and $F_{1}$ measure $=0.92$, where precision is defined as $\mathrm{tp} /(\mathrm{tp}+\mathrm{fp})$, recall $=\mathrm{tp} /(\mathrm{tp}+\mathrm{fn})$, where tp, fp, and fn mean true positive, false positive, and false negative, respectively. $F_{1}$ measure is defined as the harmonic mean of precision and recall, i.e., $2 \times$ precision $\times$ recall $/($ precision + recall $)$ [31]. Due to the imbalance between the informative and uninformative reviews in the dataset, we reported precision, recall, and $F_{1}$ measure and the fastText model performed reasonably well despite the manual work involved in labeling reviews. Then we trained the model with the 9,996 labeled review data to filter the uninformative reviews from the rest of the data, and this process removed 19,800 (21.58\%) reviews from the original dataset.

\section{CUSTOMER NEEDS TOPIC MODELING USING LDA}

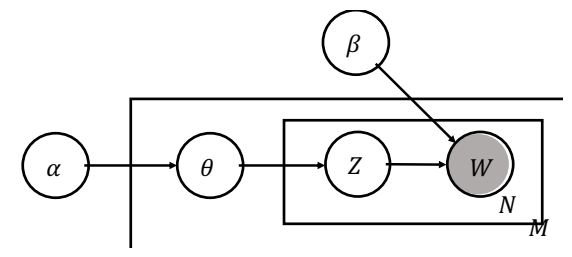

FIGURE 3: REPRESENTATION OF THE LDA MODEL
LDA is an unsupervised topic modeling technique used to cluster informative customer reviews into separate groups [20]. The LDA model is illustrated in Figure 3, where the boxes represent repeated entities. Those in white circles represent latent variables and only words in the shaded circle are the observed variables. The outer box shows the number (i.e., $M=71938$ ) of the informative customer reviews extracted using fastText and the inner box represents the number of words (i.e., $N$ ) in the customer reviews. Thus, each review is represented as a sequence of $N$ word, $\mathbf{w}=\left(w_{1}, w_{2}, \ldots, w_{N}\right)$ and each word is represented as a one-hot vector in $V$ dimension, where $V$ is the total number of the words in all the reviews, and only the $v-t h$ word is 1 if it shows up in the review. A description of the generated process is shown below:

for each document

draw a Dirichlet prior topic distribution from $\boldsymbol{\theta} \sim \operatorname{Dir}(\boldsymbol{\alpha})$

$$
\begin{aligned}
& \text { for } n \in\{1, \ldots, N\} \\
& \quad \text { draw a topic distribution from } \\
& z_{n} \sim \operatorname{Multinomial}(\boldsymbol{\theta}) \\
& \quad \text { draw a word distribution from } \\
& w_{n} \sim \operatorname{Multinomial}\left(w_{n} \mid z_{n}, \boldsymbol{\beta}\right) \text { conditioned on the } \\
& \text { topic } z_{n} \text { and word probability matrix } \boldsymbol{\beta}_{K \times V}, \text { where } \\
& \beta_{k v}=p\left(w^{v}=1 \mid z^{k}=1\right) \\
& \text { end for }
\end{aligned}
$$

\section{end for}

Based on the above process, $\boldsymbol{\theta}$ is a $K$-dimensional Dirichlet random vector of probabilities, which must sum to 1 . The probability density

$$
p(\boldsymbol{\theta} \mid \boldsymbol{\alpha})=\frac{\Gamma\left(\sum_{i=1}^{K} \alpha_{i}\right)}{\prod_{i=1}^{K} \alpha_{i}} \theta_{1}^{\alpha_{1}-1} \ldots \theta_{K}^{\alpha_{K}-1}
$$

where $\boldsymbol{\alpha}$ is a $K$ dimensional vector of positive reals and $\Gamma(x)$ is the Gamma function. We can write the joint distribution of a topic mixture $\boldsymbol{\theta}, N$ topics $\boldsymbol{z}$, and $N$ words $\boldsymbol{w}$, conditioned on the given parameters $\boldsymbol{\alpha}$ and $\boldsymbol{\beta}$

$$
p(\boldsymbol{\theta}, \boldsymbol{z}, \boldsymbol{w} \mid \boldsymbol{\alpha}, \boldsymbol{\beta})=p(\boldsymbol{\theta} \mid \boldsymbol{\alpha}) \prod_{n=1}^{N} p\left(z_{n} \mid \boldsymbol{\theta}\right) p\left(w_{n} \mid z_{n}, \boldsymbol{\beta}\right) .
$$


TABLE 1: EXTRACTED TOPICS AND THEIR ASSOCIATED STEMMED TOPIC WORDS USING LDA

\begin{tabular}{|c|c|c|c|c|c|c|c|c|c|c|c|}
\hline Topics I & Entertainment & $\begin{array}{l}\text { Great } \\
\text { Value }\end{array}$ & Interaction & $\begin{array}{l}\text { Music- } \\
\text { related }\end{array}$ & $\begin{array}{c}\text { Parental } \\
\text { control }\end{array}$ & $\begin{array}{c}\text { Cost- } \\
\text { effective }\end{array}$ & Storage & Hardware & Gift & $\begin{array}{l}\text { Smart } \\
\text { Home }\end{array}$ & $\begin{array}{l}\text { Turning } \\
\text { Page }\end{array}$ \\
\hline $\begin{array}{l}\frac{n}{0} \\
0 \\
3 \\
0 \\
0 \\
0 \\
0\end{array}$ & $\begin{array}{l}\text { game } \\
\text { read } \\
\text { plai } \\
\text { book } \\
\text { watch } \\
\text { movi } \\
\text { great } \\
\text { tablet } \\
\text { email } \\
\text { web } \\
\end{array}$ & $\begin{array}{l}\text { great } \\
\text { price } \\
\text { tablet } \\
\text { good } \\
\text { product } \\
\text { work } \\
\text { bui } \\
\text { kid } \\
\text { valu } \\
\text { best } \\
\end{array}$ & $\begin{array}{l}\text { time } \\
\text { work } \\
\text { get } \\
\text { everi } \\
\text { app } \\
\text { devic } \\
\text { need } \\
\text { dai } \\
\text { just } \\
\text { wifi }\end{array}$ & $\begin{array}{c}\text { music } \\
\text { alexa } \\
\text { plai } \\
\text { voic } \\
\text { great } \\
\text { amazon } \\
\text { echo } \\
\text { listen } \\
\text { command } \\
\text { love }\end{array}$ & $\begin{array}{c}\text { kid } \\
\text { great } \\
\text { tablet } \\
\text { parent } \\
\text { love } \\
\text { control } \\
\text { easi } \\
\text { user } \\
\text { entertain } \\
\text { friendli } \\
\end{array}$ & $\begin{array}{c}\text { tablet } \\
\text { recommend } \\
\text { good } \\
\text { worth } \\
\text { monei } \\
\text { well } \\
\text { get } \\
\text { great } \\
\text { price } \\
\text { look } \\
\end{array}$ & $\begin{array}{l}\text { memori } \\
\text { card } \\
\text { storag } \\
\text { expand } \\
\text { fast } \\
\text { app } \\
\text { space } \\
\text { add } \\
\text { ad } \\
\text { need }\end{array}$ & $\begin{array}{c}\text { good } \\
\text { great } \\
\text { qualiti } \\
\text { sound } \\
\text { speaker } \\
\text { pictur } \\
\text { screen } \\
\text { batteri } \\
\text { life } \\
\text { camera }\end{array}$ & $\begin{array}{l}\text { bought } \\
\text { love } \\
\text { gift } \\
\text { christma } \\
\text { purchas } \\
\text { got } \\
\text { tablet } \\
\text { daughter } \\
\text { wife } \\
\text { son } \\
\end{array}$ & $\begin{array}{l}\text { echo } \\
\text { home } \\
\text { light } \\
\text { control } \\
\text { smart } \\
\text { devic } \\
\text { hous } \\
\text { alexa } \\
\text { room } \\
\text { work }\end{array}$ & $\begin{array}{c}\text { page } \\
\text { turn } \\
\text { screen } \\
\text { get } \\
\text { like } \\
\text { time } \\
\text { button } \\
\text { back } \\
\text { read } \\
\text { down } \\
\end{array}$ \\
\hline opics & Reading & $\begin{array}{c}\text { Buying } \\
\text { Experience }\end{array}$ & For Kids & Streaming & Apps & Usability & Battery & Alexa & Charging & Size & $\begin{array}{c}\text { Amazon } \\
\text { Prime }\end{array}$ \\
\hline $\begin{array}{l}\frac{n}{0} \\
0 \\
3 \\
0 \\
0 \\
0 \\
0\end{array}$ & $\begin{array}{c}\text { kindl } \\
\text { read } \\
\text { fire } \\
\text { book } \\
\text { like } \\
\text { screen } \\
\text { new } \\
\text { paperwhit } \\
\text { better } \\
\text { version }\end{array}$ & $\begin{array}{l}\text { purchas } \\
\text { bui } \\
\text { best } \\
\text { kindl } \\
\text { fire } \\
\text { amazon } \\
\text { get } \\
\text { tablet } \\
\text { bought } \\
\text { first }\end{array}$ & $\begin{array}{c}\text { old } \\
\text { year } \\
\text { love } \\
\text { bought } \\
\text { tablet } \\
\text { daughter } \\
\text { purchas } \\
\text { son } \\
\text { kindl } \\
\text { got }\end{array}$ & $\begin{array}{c}\text { fire } \\
\text { stick } \\
\text { amazon } \\
\text { stream } \\
\text { box } \\
\text { fast } \\
\text { faster } \\
\text { better } \\
\text { work } \\
\text { devic }\end{array}$ & $\begin{array}{c}\text { app } \\
\text { amazon } \\
\text { store } \\
\text { game } \\
\text { download } \\
\text { googl } \\
\text { plai } \\
\text { access } \\
\text { free } \\
\text { avail }\end{array}$ & $\begin{array}{c}\text { easi } \\
\text { set } \\
\text { setup } \\
\text { learn } \\
\text { fun } \\
\text { navig } \\
\text { simpl } \\
\text { great } \\
\text { work } \\
\text { user }\end{array}$ & $\begin{array}{l}\text { read } \\
\text { light } \\
\text { batteri } \\
\text { easi } \\
\text { great } \\
\text { life } \\
\text { screen } \\
\text { kindl } \\
\text { long } \\
\text { last }\end{array}$ & $\begin{array}{c}\text { music } \\
\text { ask } \\
\text { plai } \\
\text { weather } \\
\text { alexa } \\
\text { question } \\
\text { new } \\
\text { answer } \\
\text { get } \\
\text { love }\end{array}$ & $\begin{array}{c}\text { list } \\
\text { thing } \\
\text { charg } \\
\text { just } \\
\text { like } \\
\text { need } \\
\text { charger } \\
\text { shop } \\
\text { time } \\
\text { make }\end{array}$ & $\begin{array}{c}\text { size } \\
\text { easi } \\
\text { read } \\
\text { perfect } \\
\text { small } \\
\text { book } \\
\text { light } \\
\text { carri } \\
\text { travel } \\
\text { take }\end{array}$ & $\begin{array}{c}\text { amazon } \\
\text { prime } \\
\text { cabl } \\
\text { watch } \\
\text { movi } \\
\text { show } \\
\text { stream } \\
\text { fire } \\
\text { member } \\
\text { great }\end{array}$ \\
\hline
\end{tabular}

The likelihood of an informative review document can be obtained by integrating over $\boldsymbol{\theta}$ and summing over $\boldsymbol{z}$

$$
p(\boldsymbol{w} \mid \boldsymbol{\alpha}, \boldsymbol{\beta})=\int p(\boldsymbol{\theta} \mid \boldsymbol{\alpha})\left(\prod_{n=1}^{N} \sum_{z_{n}} p\left(z_{n} \mid \boldsymbol{\theta}\right) p\left(w_{n} \mid z_{n}, \boldsymbol{\beta}\right)\right) d \boldsymbol{\theta}
$$

Finally, the topic mixture $\boldsymbol{\theta}$ can be obtained using the posterior distribution of the hidden variables of a review document as follows:

$$
p(\boldsymbol{\theta}, \boldsymbol{z} \mid \boldsymbol{w}, \boldsymbol{\alpha}, \boldsymbol{\beta})=\frac{p(\boldsymbol{\theta}, \boldsymbol{z}, \boldsymbol{w} \mid \boldsymbol{\alpha}, \boldsymbol{\beta})}{p(\boldsymbol{w} \mid \boldsymbol{\alpha}, \boldsymbol{\beta})} .
$$

However, it is intractable to obtain the analytical solution of the parameter inference of the LDA model. Various estimate methods have been proposed, including Gibbs sampling [32] and variational methods [15]. In this research, we made use of the Text Analytics Toolbox in Matlab 2017b for parameter estimation.
In order to evaluate the LDA model and to determine the number of topics, a perplexity measure was used. After training the LDA model, the perplexity of the test dataset was calculated to measure how well the model can predict the test data. A low perplexity score indicates a better prediction performance [19]. The perplexity is defined as follows:

$$
\operatorname{Perplexity}\left(D_{\text {test }}\right)=\exp \left(-\frac{\sum_{m=1}^{M} \log p\left(\boldsymbol{w}_{\boldsymbol{m}}\right)}{\sum_{m=1}^{M} N_{m}}\right),
$$

where $N_{m}$ is the total number of words in the $m-t h$ review document in the test dataset $D_{\text {test }}$ with $M$ review documents and $10 \%$ of the data were randomly selected as the test dataset. Figure 4 shows how the perplexity measure changes when the number of topics varies from 8 to 30 with a step size of 2 . The minimum value of perplexity was reached when the number of topics was 22 as shown in Figure 4. After these topics were generated, we manually assign a name to each group. 

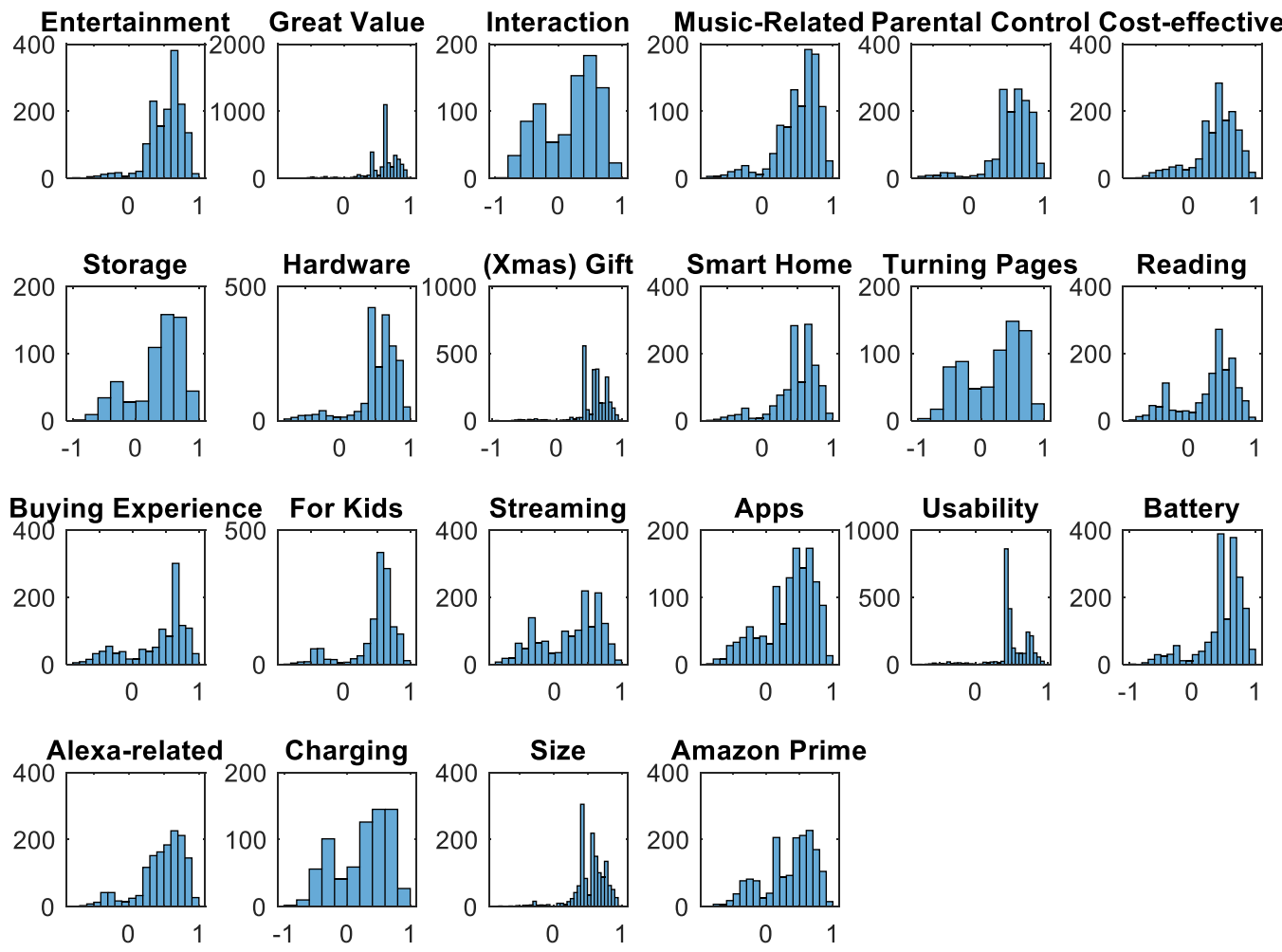

\section{FIGURE 5: HISTOGRAMS OF THE SENTIMENT INTENSITY OF THE DIFFERENT CUSTOMER REVIEWS TOPICS}

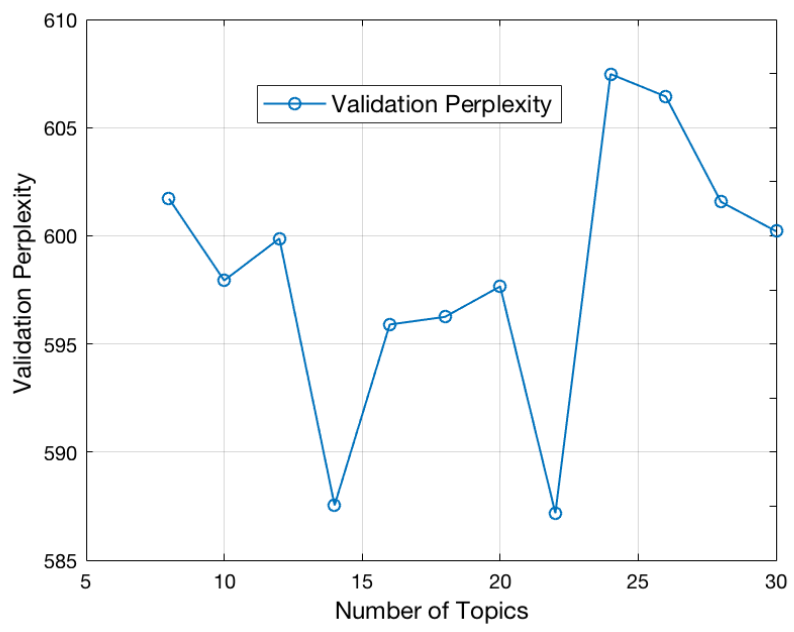

FIGURE 4: HOW THE PERPLEXITY MEASURE CHANGES WITH THE NUMBER OF TOPICS

Furthermore, we set up a threshold of topic probability in order to exclude ambiguous reviews and it was set as one standard deviation below the mean among the maximum probabilities of all the reviews predicted by the LDA model. Then we ended up with 60565 informative reviews and 22 topics as shown in Table 1 (Note the words were stemmed). The extracted topics cover various products and services within the Amazon product ecosystems. The topic "Entertainment" is most relevant to Kindle Fire HD tablets, the topic "Reading" is most relevant to Kindle E-reader, and the topic "Alexa" is most relevant to Amazon Echo and Echo Dot products. Some topics cover more than one product or service. For example, the topic "Amazon Prime" covers all the topics related to the prime service, the topics "Storage" and "Battery" are relevant to both Kindle Fire HD tablets and Kindle E-readers, and the topic "Streaming" is relevant to Fire TV, Kindle Fire HD tablets, and the Amazon Prime service. In terms of interpreting the topics, we examined not only the most relevant topic words associated with these topics as shown in Table 1, but also example reviews with high probabilities. Some topics are easier to understand while others tend to be difficult to interpret. For example, the topic "Storage" is easy to interpret while the topic "Interaction" tends to be difficult without examining review examples (e.g., "trying to type an email and the keyboard would just stop working").

\section{CUSTOMER SATISFACTION ANALYSIS WITH VADER}

VADER is a lexicon and rule-based sentiment analysis technique used to predict the sentiment and its intensity of the customer review. Hutto and Gilbert [9] showed that VADER performance was similar to human raters based on the correlation coefficient $(r=0.88)$, but regarding the classification accuracy of social media data, the performance was better than human raters. In VADER, over 9000 affective lexicons were used, including word banks and social media slangs. In order to predict the intensity of the lexical features, a systematic control process was 
used. First, ten Amazon Mechanical Turk workers, who went through a rigorous training and selection process, rated the lexicons in a range from 4 (most positive valence) to -4 (most negative valence). Then the intensity of the review was modified using five generalizable heuristics, including punctuation emphasis, capitalization differentiation, degree intensifiers, contrastive conjunction, and tri-grams before affective lexicons in the data. By averaging and normalizing the affective lexicon scores between -1 and 1 , the overall intensity of the reviews was calculated, where those smaller than 0 are negative and those larger than 0 are positive. The larger the absolute value, the more intense the sentiment is. For example, the review "this product so far has not disappointed" was predicted as 0.3724 , and the review "not easy for elderly users cease of ads that pop up" was predicted as -0.3412 .

Of all the 60565 informative reviews, $68.3 \%$ were predicted to be positive, $21.8 \%$ neutral, and $9.9 \%$ negative. All the 13199 neutral reviews were removed, and the rest was used for the following analysis. The histograms of the normalized sentiment intensity of the customer review topics are shown in Figure 5, representing the distribution of satisfaction (between 0 and 1) and dissatisfaction (between -1 and 0 ) of customers with the Amazon product ecosystem. Each histogram shows how well the customer needs are satisfied by their corresponding products and/or services in the Amazon product ecosystem. For example, the histograms of "Entertainment", "Music-Related", "ParentalControl", "Cost-effective", "Hardware", "(Xmas) Gift", "Smart Home", "For Kids", "Battery", and "Size" show that most of the reviews were positively evaluated. While almost an equal number of positive and negative reviews were seen for the following topics: "Interaction", "Turning Pages", "Streaming", and "Charging".

\section{CUSTOMER NEEDS CATEGORIZATION WITH THE ANALYTIC KANO MODEL}

An extension to the traditional Kano model was used in this paper, i.e., the analytical Kano model, to categorize customer needs using the extracted topics of product reviews and the sentiment intensity values, indicating quantitative customer preferences. The categorization process is based on the level of customer satisfaction and dissatisfaction derived from the sentiment analysis. The results are shown in Figure 6, where the $\mathrm{x}$-axis and $\mathrm{y}$-axis represent the normalized dissatisfaction and satisfaction levels of the 22 topics, respectively. The levels of satisfaction and dissatisfaction are calculated using the sentiment intensity and the number of positive and negative reviews as shown below [20]:

$$
\begin{gathered}
X_{i}=\lambda \times \frac{N R_{i}}{T R_{i}} \times I N R_{i}, \\
Y_{i}=\frac{P R_{i}}{T R_{i}} \times I P R_{i},
\end{gathered}
$$

where $\lambda$ indicates the degree of dissatisfaction, $N R_{i}, P R_{i}$, and $T R_{i}$ represent the total numbers of negative, positive, and total reviews and $I N R_{i}, I P R_{i}$, are the mean intensity values of the negative and positive reviews, respectively. The customer need is represented as a vector $\boldsymbol{r}_{\boldsymbol{i}}$ of magnitude $r_{i}$ and direction $\alpha_{i}$, where $\quad 0 \leq r_{i}=\left|\boldsymbol{r}_{\boldsymbol{i}}\right|=\sqrt{X_{i}^{2}+Y_{i}^{2}} \leq \sqrt{2} \quad$ and
$0 \leq \alpha_{i}=\arctan \left(Y_{i} / X_{i}\right) \leq \pi / 2$ is the angle between the horizontal axis and $\boldsymbol{r}_{\boldsymbol{i}}$. Four types of customer needs were identified based on the Kano model: (1) attractive needs that are unexpected by the customer and can increase his/her satisfaction substantially, (2) one-dimensional needs that linearly increase customer satisfaction, (3) must-be needs that are expected by the customer and lead to dissatisfaction if they are not satisfied, and (4) indifferent needs that the customer is not intrested in. The attractive needs are shown in the area AIHB in Figure 6(a) when $r_{i} \geq r_{0}$ and $\alpha_{H} \leq \alpha_{i} \leq \pi / 2$; the one-dimensional needs lie in the area HBCDG in Figure 6(a) when $r_{i} \geq r_{0}$ and $\alpha_{L} \leq \alpha_{i} \leq \alpha_{H}$; the must-be needs are shown in the area GDEF in Figure 6(a) when $r_{i} \geq r_{0}$ and $0 \leq \alpha_{i} \leq \alpha_{L}$; the indifferent needs lie in the area OIF in Figure 6(a) when $r_{i}<r_{0}$. In this paper, the following parameters are assumed based on a sensitivety analysis to illustrate the performance of the Amazon product ecosystem: $\lambda=3, \quad r_{0}=0.4, \alpha_{L}=\pi / 6, \quad$ and $\alpha_{H}=\pi / 3$.

Figure 6(a) and Figure 6(b) represent the classification results in terms of the different topics of customer needs for the collected reviews before January 1, 2015 and for all the reviews collected, respectively. The blue plus signs represent attractive needs, the red crosses represent one-dimensional needs, the purple circles represent indifferent needs, and the green asterisks represent must-be needs. It is noticed that the customer needs change with time. For instance, the topic "Cost-effective" has changed from a one-dimensional customer need in Figure 6(a) to an attractive need in Figure 6(b). The topic "Smart Home" has changed from an indifferent customer need in Figure 6(a) to an attractive customer need in Figure 6(b). The topic "Charging" has changed from a must-be customer need in Figure 6(a) to a one-dimensional customer need in Figure 6(b). From Figure 6(b), we can tell that all the customer needs are either onedimensional or attractive. By examining the review data at different times, i.e., different stages of the product ecosystem, it shows the dynamic evolution of the product ecosystem, which is helpful to understand the evolution of different customer needs within the.

The paramteres involved in the analytical Kano model can also be adjusted to show how they influence the results. The aversion of customer dissatisfaction is often around 2.5 to 3.5. The increase of $\lambda$ was shown to increase the degree of customer dissatisfaction. The range of $r_{0}$ is often between 0.3 to 0.5 , it was noticed that if $r_{0}$ is 0.5 , some of the topics (i.e., "Interaction", "Cost-effective", "Storage", "Reading", "Streaming", "Apps", "Charging", and "Amazon Prime") will be considered indifferent which is not reasonable. As long as $r_{0}$ is less than 0.4437 (minimum value of $r_{0}$ for topic 22) the results in Figure 6 (b) will not change. To study the influence of $\alpha_{L}$ and $\alpha_{H}$, we varied their values between $\pi / 12$ to $\pi / 4$ and $\pi / 4$ to $5 \pi / 12$ respectively [30]. By increasing the value of $\alpha_{L}$, the topic "Turning pages" has changed from being a one-dimensional to a must-be need. And by increasing $\alpha_{H}$, some topics (i.e., "Interaction", "Cost-effective", "Storage", "Reading", "Buying Experience", "Charging", and "Amazon Prime") have changed from being attractive to one-dimensional needs, which makes more sense. 


\section{DISCUSSION}

1) Reducing ambiguity: In this study, we analyzed customer needs of the Amazon product ecosystem using online product reviews. The first step was to remove uninformative reviews using fastText, where we manually labeled 9996 reviews into informative and uninformative reviews. During the topic modeling, 11373 reviews were removed, since they had low probabilities for the 22 topics. Then, for the purpose of evaluating the level of satisfaction and dissatisfaction, all the 13199 neutral reviews were removed. Hence, 47366 reviews were used to classify customer needs in the analytical Kano model. Such a multi-step filtering process greatly improved data quality, reliability, and validity of the results.

2) Improving efficiency and effectiveness of data analysis: In order to analyze a large volume of text data, we made use of three machine learning techniques, including fastText, LDA, and VADER. The fastText is a supervised machine learning technique used to remove uninformative reviews . To improve the performance of noise filtering, we had to manually label customer reviews to train and validate the model. Compared to traditional coding in text data analysis in the domain of customer needs elicitation and analysis, it was much easier and more efficient to label the text data here in order to build the fastText model, as it only involved two categories, i.e., informative or not. Second, both LDA and VADER are unsupervised machine learning techniques, and thus no human manual work was involved. Compared with traditional customer needs analysis methods, machine learning methods not only improve the efficiency of the data analysis, but also are likely to produce more valuable customer needs from online product reviews [13].

3) Categorizing customer needs: In order to analyze customer needs within the Amazon product ecosystem, we first made use of the satisfaction and dissatisfaction scores produced from the sentiment analysis step, which gave us a tangible criterion in categorizing different types of customer needs and in measuring the performance of the product ecosystem. Specific products and services associated with these customer needs can be further examined in order to further improve user experience and satisfaction of the product ecosystem. Second, we examined the customer needs along the temporal dimension using the analytical Kano model, which showed the evolution of the customer needs within the Amazon product ecosystem. However, it was also observed that certain customer needs (and their associated product attributes) did not follow the product life cycle suggested by Kano [22] where the product state changes from indifferent to attractive, to one-dimensional, and finally to must-be. Two possible reasons can be identified. First, we only had a small amount of data (493 reviews) before Jan. 1, 2015 and fewer products and services were involved in the product ecosystem, which might produce less reliable results. Second, it is still too short a time (from 2011 to 2018) to show the full life cycle of the product ecosystem. For example, it took 18 years from the remote control to change from an attractive customer need (i.e., 1983) to a must-be one (i.e., 1998) [33].

Furthermore, in order to understand the customer needs within the product ecosystem, we need to study different

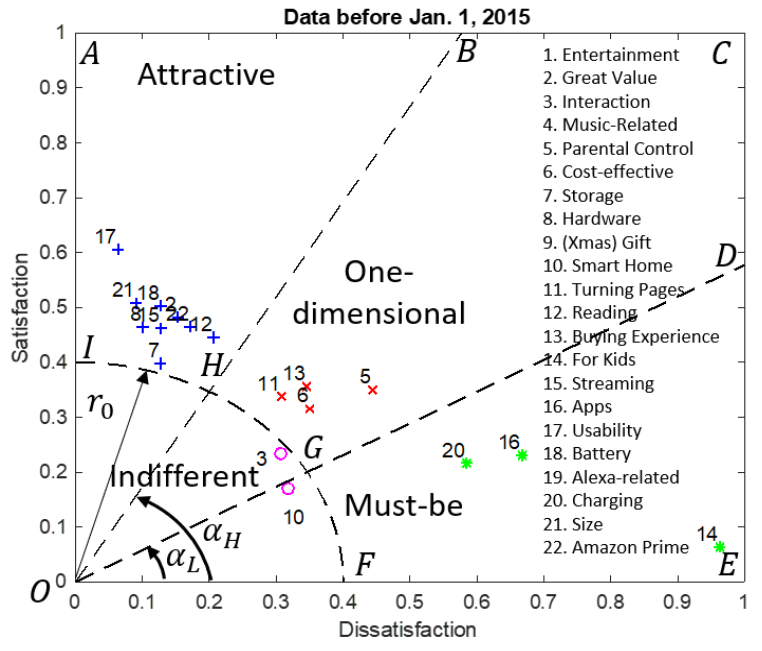

(a)

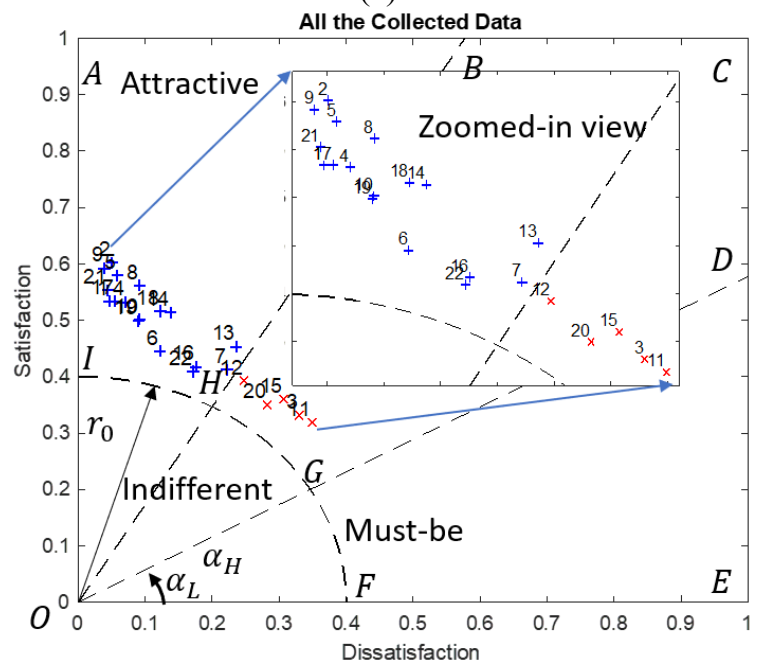

(b)

\section{FIGURE 6: CUSTOMER NEEDS CATEGORIZATION USING ANALYTICAL KANO MODEL (a) USING DATA BEFORE JAN. 1, 2015 AND (b) USING ALL THE COLLECTED DATA}

characteristics and relations of the products and services. By making use of the analytical Kano model, we can see that "Amazon Prime" was considered as an attractive need since it can provide many services (i.e. product discounts, free 2 days shipping, free video streaming, and free eBooks...). And these services can positively affect other topics, such as "Cost Effective", "Entertainment", "Music-Related", "Reading", "Streaming", "Alexa-related" and "Buying Experience". Hence, understanding the relationships between the products and services of the ecosystem is essential for creating desired user experience.

4) Limitation and future work: The first limitation of this study is related to the unbalanced distribution of data over the time. The data before 2015 can be unreliable as compared to after 2015 since we only had 493 reviews. Therefore, more data should be collected before 2015 in order to show a complete picture of the product ecosystem. Second, showing the full life 
cycle of a product ecosystem can provide more reliable and precise analysis. For example, understanding the evolution of "Smart Home" over the time, from the day it was created till now, can help us understand why the customer need was changed from being indifferent before 2015 to be attractive in 2018 (see Figure 6). The topic "Smart Home" is most relevant to Alexa products and services and it was first launched in November 2014. Therefore, it seems that the evolution of this customer need makes sense. However, at the current stage, more data in the future are needed in order to show the full life cycle of the product ecosystem. Third, further investigations should be conducted to understand the needs for individual products and services and their roles and interrelationships in the whole product ecosystem.

\section{CONCLUSIONS}

In this work, we analyzed the customer needs of the Amazon product ecosystem by making use of online product reviews. Using both supervised (fastText) and unsupervised (LDA and VADER) machine learning techniques, we were able to reduce ambiguity involved in the text data and analyze customer needs for products and services involved in the product ecosystem effectively and efficiently. fastText was used to filter the noise from the online customer reviews. LDA was used to cluster the customer needs into different topics. VADER helped in recognizing customer preferences quantitatively regarding different products and services. Finally, the analytical Kano model was used to categorize customer needs based on satisfaction and dissatisfaction scores. The proposed method was demonstrated using the Amazon product ecosystem and the process and the results showed the potential of the proposed method.

\section{REFERENCES}

[1] Zhou, F., Xu, Q., and Jiao, R. J., 2011, "Fundamentals of Product Ecosystem Design for User Experience," Res. Eng. Des., 22(1), pp. 43-61.

[2] Miguel, J. C., and Casado, M. Á., 2016, "GAFAnomy (Google, Amazon, Facebook and Apple): The Big Four and the B-Ecosystem," Dynamics of Big Internet Industry Groups and Future Trends, pp. 127-148.

[3] Levin, M., 2014, Designing Multi-Device Experiences: An Ecosystem Approach to User Experiences Across Devices, "O’Reilly Media, Inc."

[4] Gawer, A., and Cusumano, M. A., 2014, "Industry Platforms and Ecosystem Innovation," Journal of Product Innovation Management, 31(3), pp. 417-433.

[5] Jiao, R. J., Xu, Q., Du, J., Zhang, Y., Helander, M., Khalid, H. M., Helo, P., and Ni, C., 2007, "Analytical Affective Design with Ambient Intelligence for Mass Customization and Personalization," Int. J. Flexible Manuf. Syst., 19(4), pp. 570-595.

[6] Oh, H. S., Moon, S. K., and Kim, W., 2012, "A ProductService System Design Framework Based on a Business Ecosystem," ASME 2012 International Design Engineering Technical Conferences and Computers and
Information in Engineering Conference, American Society of Mechanical Engineers, pp. 1033-1042.

[7] Zhou, F., Jiao, R. J., Xu, Q., and Takahashi, K., 2012, "User Experience Modeling and Simulation for Product Ecosystem Design Based on Fuzzy Reasoning Petri Nets," IEEE Transactions on Systems, Man, and Cybernetics Part A: Systems and Humans, 42(1), pp. 201-212.

[8] Crandall, B., Klein, G. A., and Hoffman, R. R., 2006, Working Minds: A Practitioner's Guide to Cognitive Task Analysis, MIT Press.

[9] Wang, Y., Mo, D. Y., and Tseng, M. M., 2018, "Mapping Customer Needs to Design Parameters in the Front End of Product Design by Applying Deep Learning," CIRP Ann., 67(1), pp. 145-148.

[10] Zhou, F., Jiao, R. J., and Linsey, J. S., 2015, "Latent Customer Needs Elicitation by Use Case Analogical Reasoning from Sentiment Analysis of Online Product Reviews," J. Mech. Des., 137(7), p. 071401.

[11] Bickart, B., and Schindler, R. M., 2001, "Internet Forums as Influential Sources of Consumer Information," Journal of Interactive Marketing, 15(3), p. 31.

[12] Tseng, M. M., and Jiao, J., 1998, "Computer-Aided Requirement Management for Product Definition: A Methodology and Implementation," Concurrent Eng.: Res. Appl., 6(2), pp. 145-160.

[13] Timoshenko, A., and Hauser, J. R., 2017, "Identifying Customer Needs from User-Generated Content," SSRN Electronic Journal.

[14] Joulin, A., Grave, E., Bojanowski, P., and Mikolov, T., 2017, "Bag of Tricks for Efficient Text Classification," Proceedings of the 15th Conference of the European Chapter of the Association for Computational Linguistics: Volume 2, Short Papers.

[15] Blei, D. M., Ng, A. Y., and Jordan, M. I., 2003, "Latent Dirichlet Allocation," J. Mach. Learn. Res., 3(Jan), pp. 993-1022.

[16] Wang, W., Feng, Y., and Dai, W., 2018, “Topic Analysis of Online Reviews for Two Competitive Products Using Latent Dirichlet Allocation," Electron. Commer. Res. Appl., 29, pp. 142-156.

[17] Liu, B., 2010, "Sentiment Analysis and Subjectivity," Handbook of natural language processing, 2, pp. 627-666.

[18] Hu, M., and Liu, B., 2004, "Mining and Summarizing Customer Reviews," Proceedings of the 2004 ACM SIGKDD International Conference on Knowledge Discovery and Data Mining - KDD '04.

[19] Ding, X., Liu, B., and Yu, P. S., 2008, “A Holistic LexiconBased Approach to Opinion Mining," Proceedings of the International Conference on Web Search and Web Data Mining - WSDM '08.

[20] Chen, L., Qi, L., and Wang, F., 2012, "Comparison of Feature-Level Learning Methods for Mining Online Consumer Reviews," Expert Syst. Appl., 39(10), pp. 95889601.

[21] Kim, Y., 2014, "Convolutional Neural Networks for Sentence Classification," Proceedings of the 2014 Conference on Empirical Methods in Natural Language Processing (EMNLP), Association for Computational Linguistics, Stroudsburg, PA, USA, pp. 1746-1751. 
[22] Li, Z., Zhang, Y., Wei, Y., Wu, Y., and Yang, Q., 2017, "End-to-End Adversarial Memory Network for CrossDomain Sentiment Classification," Proceedings of the Twenty-Sixth International Joint Conference on Artificial Intelligence.

[23] Hutto, C. J., and Gilbert, E., 2014, "Vader: A Parsimonious Rule-Based Model for Sentiment Analysis of Social Media Text," Eighth International Conference on Weblogs and Social Media (ICWSM-14).

[24] Zhou, F., Ji, Y., and Jiao, R. J., 2012, "Affective and Cognitive Design for Mass Personalization: Status and Prospect,” J. Intell. Manuf., 24(5), pp. 1047-1069.

[25] Kano, N., 1984, "Attractive Quality and Must-Be Quality," Hinshitsu (Quality, The Journal of Japanese Society for Quality Control), 14, pp. 39-48.

[26] Kim, J., and Han, S. H., 2008, "A Methodology for Developing a Usability Index of Consumer Electronic Products,” Int. J. Ind. Ergon., 38(3-4), pp. 333-345.

[27] Delin, J., Sharoff, S., Lillford, S., and Barnes, C., 2007, "Linguistic Support for Concept Selection Decisions," Artif. Intell. Eng. Des. Anal. Manuf., 21(02).

[28] Wassenaar, H. J., Chen, W., Cheng, J., and Sudjianto, A., 2005, "Enhancing Discrete Choice Demand Modeling for Decision-Based Design,” J. Mech. Des., 127(4), p. 514.

[29] Zhou, F., Ji, Y., \& Jiao, R. J. (2015). "Prospect Theoretic Modeling of Customer Affective - Cognitive Decisions under Uncertainty for User Experience Design".

[30] Xu, Q., Jiao, R. J., Yang, X., Helander, M., Khalid, H. M., and Opperud, A., 2009, "An Analytical Kano Model for Customer Need Analysis," Design Studies, 30(1), pp. 87110.

[31] Zhou, F., Qu, X., Helander, M. G., and Jiao, J. (roger), 2011, "Affect Prediction from Physiological Measures via Visual Stimuli," Int. J. Hum. Comput. Stud., 69(12), pp. 801-819.

[32] Griffiths, T. L., and Steyvers, M., 2004, "Finding Scientific Topics," Proceedings of the National Academy of Sciences, 101(Supplement 1), pp. 5228-5235.

[33] Kano, S., 2001, "Life Cycle and Creation of Attractive Quality," Proceedings of the 4th International Quality Management and Organisational Development Conference, pp. 18-36. 